\title{
A multiple streamline approach to high angular resolution diffusion tractography
}

\author{
Yi-Ping Chao ${ }^{\mathrm{a}}$, Jyh-Horng Chen ${ }^{\mathrm{a}}$, Kuan-Hung Cho ${ }^{\mathrm{a}}$, Chun-Hung $\mathrm{Yeh}^{\mathrm{b}}$, \\ Kun-Hsien Chou ${ }^{c}$, Ching-Po Lin ${ }^{b, d, *}$ \\ ${ }^{a}$ Institute of Electrical Engineering, National Taiwan University, Taipei, Taiwan \\ ${ }^{\mathrm{b}}$ Institute of Biomedical Imaging and Radiological Sciences, National Yang Ming University, Taipei, Taiwan \\ ${ }^{\mathrm{c}}$ Institute of Biomedical Engineering, National Yang Ming University, Taipei, Taiwan \\ ${ }^{\mathrm{d}}$ Institute of Neuroscience, National Yang Ming University, Taipei, Taiwan
}

Received 30 June 2007; received in revised form 16 January 2008; accepted 28 January 2008

\begin{abstract}
Diffusion-weighted magnetic resonance imaging has the ability to map neuronal architecture by estimating the 3D diffusion displacement within fibrous brain structures. This approach has non-invasively been demonstrated in the human brain with diffusion tensor tractography. Despite its valuable application in neuroscience and clinical studies however, it faces an inherent limit in mapping fiber tracts through areas with intervoxel incoherence. Recent advances in high angular resolution diffusion imaging have surpassed this limit and have the ability to resolve the complex fiber intercrossing within each MR voxel. To connect the fiber tracts from a multi-fiber system, this study proposed a modified fiber assignment using the continuous tracking (MFACT) algorithm and a tracking browser to propagate tracts along complex diffusion profiles. The Q-ball imaging method was adopted to acquire the diffusion displacements. Human motor pathways with seed points from the internal capsule, motor cortex, and pons were studied respectively. The results were consistent with known anatomy and demonstrated the promising potential of the MFACT method in mapping the complex neuronal architecture in the human brain.
\end{abstract}

(C) 2008 IPEM. Published by Elsevier Ltd. All rights reserved.

Keywords: High angular resolution diffusion imaging; QBI; Tractography; MRI

\section{Introduction}

Diffusion-weighted magnetic resonance imaging (DWMRI) is a non-invasive method used for mapping the neuronal microstructure by estimating the 3D displacement distribution of diffusing water molecules within fibrous brain tissues. Thus, it may be employed to define complex neural circuitry in the human brain [1,2]. Diffusion tensor (DTI), one of DW-MRI techniques, demonstrates its primary capability in non-invasively mapping 3D reconstructions of neural systems in the rat and human brain [2,3]. Connectivity of white matter in the central nervous system, such as the corpus callosum, pyramidal tract trajectories and cingulum, have been reconstructed using the line propagation algorithm based on

\footnotetext{
* Corresponding author. Tel.: +886 228267338; fax: +8862 28262285 .

E-mail address: cplin@ym.edu.tw (C.-P. Lin).
}

the principal diffusion direction of DTI [1,4]. Despite the insufficiency of the tensor model in mapping multi-fiber directions within a single voxel, the result reveals the neural circuitry in functional studies and in patient diagnosis. However, in voxels containing fiber bundles of multiple orientations, the principal direction of the tensor model fails to determine the distribution of fibers. In human brain, one third of the regions were attributed as one fiber distribution, which might contain more than one fiber orientation [5].

A number of novel approaches have been proposed to describe the complex fiber distributions within an MR imaging voxel. These include diffusion spectrum imaging [6], Q-ball imaging (QBI) [7], the CHARMED model [8], PASMRI $[9,10]$, the deconvolution method [11,12] and multiple tensor fitting methods $[5,13-15]$. In these methods, the discrete orientations, the probability density function (PDF), the orientation distribution function (ODF), or the fiber 
orientation distribution (FOD) of anisotropic tissues were obtained to identify multiple fiber populations. Using these approaches, several tractography algorithms were implemented to denote neuronal tracts such as the superior longitudinal fasciculus and acoustic radiations. The results from multiple fiber tracking (MFT) algorithms demonstrate the potential of detecting complex fiber components and the mapping of connections in areas with complex fiber directions [5,16-18].

Despite the credible results in mapping complex neuronal connectivity, there are some intrinsic problems in existing MFT algorithms. First, tracking results are mostly constrained to regions of white matter due to the tracking stop criterion of fractional anisotropy $(\mathrm{FA})<0.2$. FA, an index of diffusion tensor, is generally less than 0.2 in a voxel with crossing fibers. Under this condition, many true-positive neuronal pathways may not be revealed by current MFT approaches [5], thus limiting the identification of gray matter connections. Second, the probability approach and the region growth method were proposed to illustrate possible connections between brain areas. However, they are very time-consuming in resolving the complexity of the diffusion pattern within each MR voxel.

In the present study, a modified MFT method called multiple fiber assignment by continuous tracking (MFACT) was proposed for tracing complex fiber trajectories. The MFACT method was applied to QBI results [7] to estimate the reticular neural connections with multiple fiber orientations. In order to improve upon the intrinsic problems of identifying falsepositive neural pathways and the lengthy time requirement, two stop criteria were applied: the angle between two discrete vectors and the length of ODF. Due to the fact that high curvature fibers do not exist in the human brain, the angle between two discrete vectors has been considered by many tractography methods in the past [1]. The length of ODF indicates the neural density along the ODF direction [19] and was first considered in this fiber tracking algorithm. Using these criteria, the MFACT method was shown to be more straightforward in mapping neural tracts and was more easily performed on a personal computer. In addition, a browser system was developed to provide the user with an interface of volume of interesting (VOI) selection of 3D MR anatomy to reveal complex brain connectivity between brain areas. According to the relationship between these brain regions and integrated with Boolean analysis, this method has the potential to identify true-positive pathways from a group of tangled tracts.

\section{Materials and methods}

\subsection{MRI acquisition}

Human brain QBI from five healthy subjects was acquired in a GE Healthcare Signa 1.5T Excite scanner (General Electric, Milwaukee, WI) with an eight-channel head coil. After alignment, placing cushions inside the coil minimized head motion of the subject. Each subject was completely informed of the study protocols by a research physician and informed consents were obtained from participants. The study was conducted in accordance with the guidelines approved by the institutional review board at Taipei Veterans General Hospital.

Isotropic axial diffusion-weighted images were obtained using a single shot diffusion spin-echo echo planar imaging (EPI) sequence with $\mathrm{TR} / \mathrm{TE}=17,000 / 91.2 \mathrm{~ms}$, $\mathrm{FOV}=256 \mathrm{~mm}$, matrix size $=128 \times 128$, yielding an image resolution of $2 \mathrm{~mm} .162$ diffusion encoding directions (4folds tessellated icosahedron) with $b$ value of $3000 \mathrm{~s} / \mathrm{mm}^{2}$ and one reference image were acquired. In order to achieve total cerebrum coverage, 46 slices with slice thickness of $2.2 \mathrm{~mm}$ were obtained. In addition, T1-weighted images were acquired for the anatomical reference with a 3D FSPGE sequence. With the same FOV as done for diffusion MRI, matrix size of 256, slice number of 124 , thickness of $1.5 \mathrm{~mm}$ and spatial resolution of $1 \mathrm{~mm}$ were yielded. The total scan time for both protocols was $60 \mathrm{~min}$.

\subsection{Q-ball imaging}

In order to measure the diffusion function and identify crossing-fibers in neural microstructures, the QBI method was performed in this study. QBI allowed multiple intravoxel fiber orientations to be constructed by applying Funk-Radon transform (FRT) on q-space signals in a 3D spherical surface. 60 points on each equator were interpolated using the RBF (Radial Basic Function) method with a Gaussian kernel from the data points located on a spherical q-space shell. Diffusion function and fiber distributions were described by ODF with 162 directions and visualized via surface rendering of 320 triangles. Finally, the estimated fiber orientations were determined by the local maximum of ODF in 3D space (Fig. 1).

\subsection{Concept of multiple fiber assignment by continuous tracking}

In order to detect neural pathways by methods that provide information regarding multiple fiber populations within all voxels, the Fiber Assignment by Continues Tracking (FACT) algorithm was modified to MFACT. FACT can be used to construct fiber trajectories by connecting the continuous and adjoining diffusion tensor with threshold conditions $[2,20]$. Initiated from manually defined seed areas or based on anatomical markers, the FACT fiber tracking starts from the center of the seed voxel and traces along bilateral directions (forward and backward) of the principal eigenvector of the diffusion tensor until each of three stop conditions was met, the FA value is less than a user-defined threshold, the tract's turning angle is larger than the user-defined threshold, and the tracing reaches the brain boundary. The MFACT algorithm extends the FACT model to multi-fiber directions within each MR voxel. It allows for the tracking of branched axonal projections at each step by dispersing along the multiple fiber 


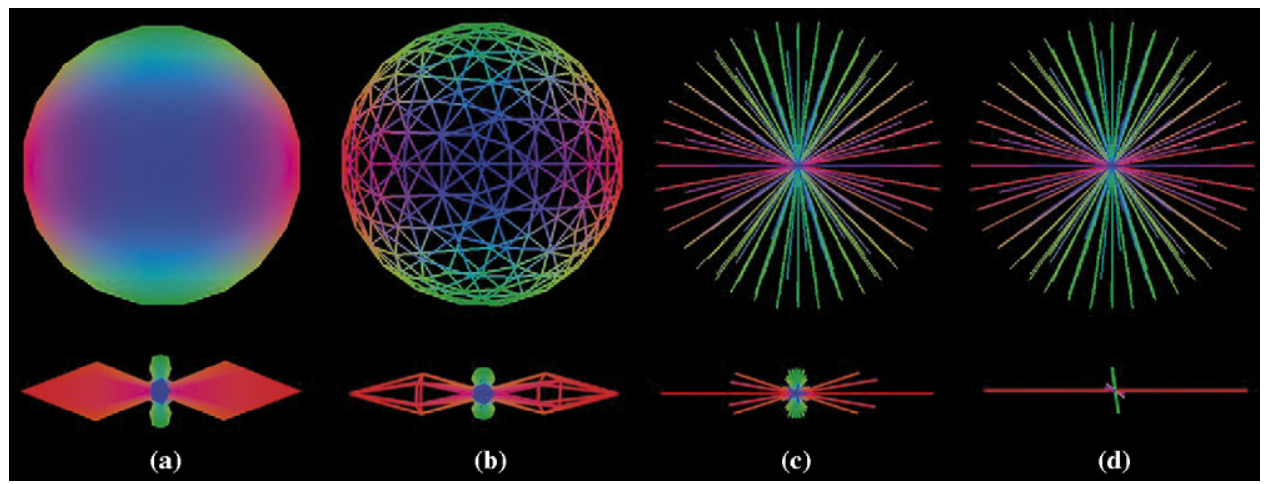

Fig. 1. Diagram of surface rendering (a), mesh (b), vector fields (c), and local maximum vector (d) of ODF from QBI.

orientations. Instead of using the FA value as the tracking criterion, the local maximum of diffusion pattern length was deemed as the criterion of propagation. As in FACT, the criterion for tract turning angles was also applied in MFACT to avoid inaccurate identification of connectivity. The detailed procedure is as follows (Fig. 2):

1. Tracking starts from the center of seed voxel $x_{i}$.

2. Take the $j$ th vector $v_{i j}$ at $x_{i}$ as the tracking direction if any $\mathrm{ODF}_{\text {lmax } \_i j}$ at the voxel $x_{i}$ is larger than the length threshold of ODF $\left(\mathrm{ODF}_{\mathrm{td}}\right)$.

3. Propagate along the vector direction $v_{i j}$ and calculate the interception point $p_{i j}$ on the boundary between voxels $x_{i}$ and $x_{i j}$.

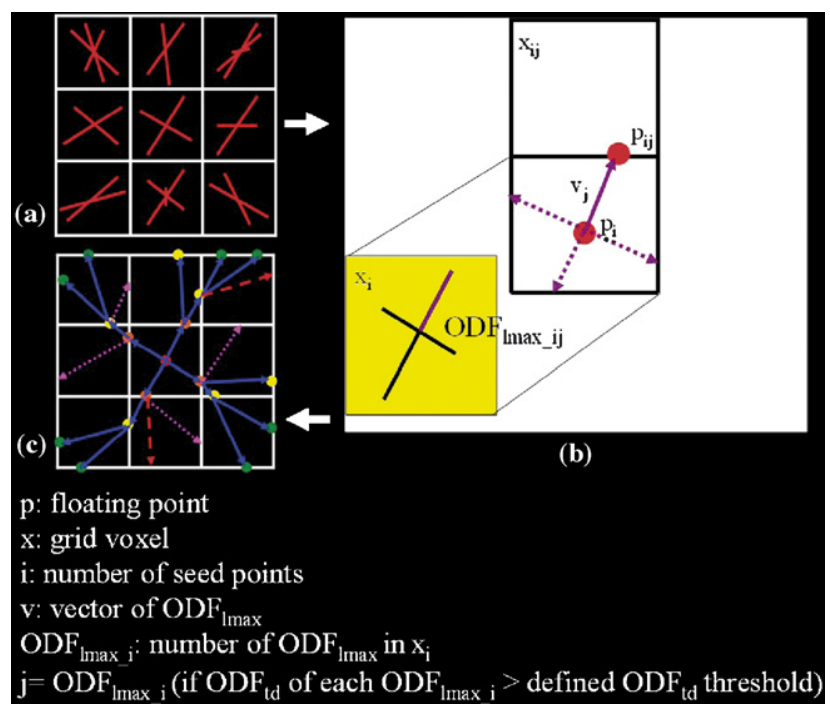

Fig. 2. Outline of the MFACT approach. Local maximum vector field of QBI can be sketched as multi lines within each MR voxel (a). Fiber tracts initiate from the center seed point, $p_{i}$, spread along the directions of the local maximum vectors, $v_{j}$, and reach the interception point $p_{i j}$ on the boundary between two voxels, $x_{i}$ and $x_{i j}$ (b). Two criteria, TTA and $\mathrm{ODF}_{\mathrm{td}}$, are used to regulate the fiber propagation (c). The plum and red dotted lines indicate the TTA and $\mathrm{ODF}_{\mathrm{td}}$ stop criterion for growing tracts, respectively. Tracts stop when the angle between two adjacent vectors is bigger than the TTA value or the length of local maximum vector is shorter than the $\mathrm{ODF}_{\mathrm{td}}$ value. The whole process is similar to a region-growing method with hierarchical red, orange, yellow, and green points which serve as seed points for further fiber tracking.
4. Assign $j+1$ as $j$ if the point $p_{i j}$ reaches the image boundary. Go to step 6 if it is not.

5. Go to step 11 if $j$ is larger than the amount of $\mathrm{ODF}_{\operatorname{lmax}}$ at voxel $x_{i}$. Go to step 2 if it is not.

6. Assign $j+1$ as $j$ if each $\mathrm{ODF}_{1 \text { max }}$ of the next voxel $x_{i j}$ is less than $\mathrm{ODF}_{\mathrm{td}}$. Go to step 8 if it is not.

7. Go to step 11 if $j$ is larger than the amount of $\mathrm{ODF}_{\operatorname{lmax}}$ at voxel $x_{i}$. Go to step 2 if it is not.

8. Calculate the angle difference between current $v_{i j}$ and each $\mathrm{ODF}_{1 \mathrm{max}}$ orientation of next voxel $x_{i j}$.

9. Assign $j+1$ as $j$ if any angle difference is larger than the tract-turning angle threshold (TTA). Go to step 11 if it is not.

10. Go to step 2 if $j$ is less than the amount of ODF $\mathrm{Imax}_{\max }$ at voxel $x_{i}$,

11. Record $p_{i j}, x_{i j}$, and the pathways from $p_{i}$ to $p_{i j}$ if the amount of $p_{i j}$ is not zero.

12. Reset all $p_{i j}$ and $x_{i j}$ as the new seed point and the seed voxel respectively, map pathway routes from $x_{i}$, and go to step 1 . Go to step 13 if it is not.

13. Apply 1-12 iteratively until all voxels of interest (VOI) are calculated.

where $x$ is the processing seed grid voxel, $\mathrm{ODF}_{1 \max }$ is the length of ODF local maximum, $\mathrm{ODF}_{\mathrm{td}}$ is $\mathrm{ODF}_{\text {lmax }}$ threshold for route trace which is the first criterion for fiber tracking, $v_{i j}$ is the $j$ th orientation of $\mathrm{ODF}_{\operatorname{lmax}}$ at voxel $v_{j}, p_{i j}$ is the interception point on the boundary between voxel $x_{i}$ and the traced voxel $x_{i j}$, and TTA is the angular criterion for tractturning within two adjacent voxels. Depending on the neural pathways and seed areas that are anticipated, the values for $\mathrm{ODF}_{\mathrm{td}}$ and TTA may be defined between 0.76 and 0.9 , and 40 and 70, respectively. A continuous neural tract is identifiable by connecting the recorded $p$ points. According to the proposed procedure, the MFACT algorithm may be regarded as a region-growing method for complex neural branches.

\subsection{VOI selection \& tract analyzer}

The interface for VOI selection was designed for several VOI assortments according to the brain markers at T1WI and T2WI, fractional anisotropy (FA), or functional images. All 
data was saved in Analyze 7.5 format after image processing to avoid time-consuming data analysis in further applications. Three anatomical views (axial, sagittal and coronal) and five background images referred to in the above section enabled the identification of brain markers as tracking seed volume. Using multi-VOI selections and Boolean functions, it was possible to identify and visualize neural tracts. Tract Analyzer was implemented to analyze the interaction between two VOIs by using Boolean functions, such as AND, OR, and NOT. The results were saved as files for further presentation and visualization. Due to the complex pathways calculated by the MFACT algorithm, the brute-force method was adopted to trace more true-positive fiber trajectories [21].

\subsection{Diffusion tensor tractography}

Diffusion tensor $\mathbf{D}$ was reconstructed from the same QBI dataset by

$\ln \left(\frac{I_{i}}{I_{0}}\right)=-\int_{0}^{\Delta} \mathbf{k}_{i}^{T}(t) \mathbf{D} \mathbf{k}_{i}(t) \mathrm{d} t$

where $I_{i}$ and $I_{0}$ represent the attenuated and non-attenuated images, respectively, $\Delta$ is the diffusion time, $\mathbf{k}_{i}(t)=$ $(2 \pi)^{-1} \int_{0}^{\Delta} \gamma \mathbf{g}_{i}(\tau) \mathrm{d} \tau$ is the spatial modulation of magnetization produced by diffusion-sensitizing gradients $\mathbf{g}_{i}$ in $i$ th direction where $i=1,2, \ldots, 162$, and $\gamma$ is the proton gyromagnetic ratio. FACT tracking algorithm with FA of 0.2 and tract-turning angle of 60 degrees was used for comparison $[2,22]$.

\section{Results}

QBI can reveal intervoxel heterogeneity within each MR voxel. Fig. 3 shows the comparison between DTI and QBI in the brain region of the centrum semiovale where three elements are intercrossed, i.e. the corticospinal tract, the corpus callosum, and the superior longitudinal fasciculus. Due to the inherent limitation of DTI, only one unique orientation can be determined by the rendering cylinder within each voxel [23] (Fig. 3a). This may induce an erroneous evaluation of the fiber orientation and consequently influence the validity of fiber propagation by tractography. In contrast, QBI has the ability to map multi-fiber incoherence within each MR voxel and therefore can reconstruct the intercrossing elements in this complex brain region. As shown in Fig. 3b, the yellow arrows indicate the primary orientations of QBI and three complex elements in the centrum semiovale can be identified (Fig. 3b).

In order to examine the capacity of the MFACT method, motor pathways were used as a reference for tractography. VOI was first defined in a healthy subject at the internal capsule, with TTA of 40 degrees and $\mathrm{ODF}_{\mathrm{td}}$ ranging from 0.76 to 0.9 , to quantify the effect of $\mathrm{ODF}_{\mathrm{td}}$ in tractography popula-

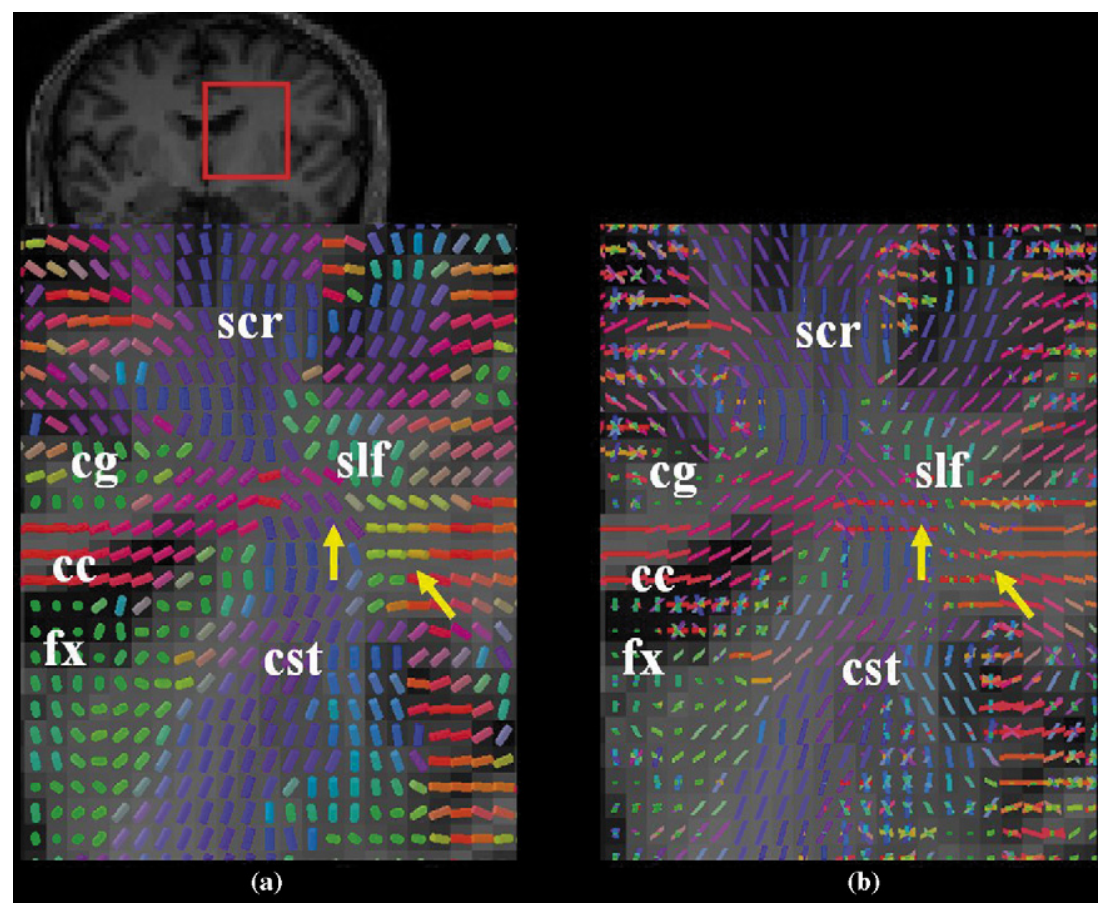

Fig. 3. Diffusion profiles in the centrum semiovale. The centrum semiovale is defined by the red rectangle on the background of T1WI. Only one cylinder is adopted to represent the primary orientation of DTI with color-coding according to its vector direction (blue: axial; red: transverse; green: anteroposterior orientation). In regions with intersecting fibers (as indicated by the yellow arrows), DTI shows only one random direction (a). On the contrary, multiple fiber populations can be identified by the local maximum vectors of ODF (b). fx: fornix, cc: corpus calloum, cst: corticospinal tract, slf: superior longitudinal fasciculus, cg: cingulum, and scr: superior corona radiata. 


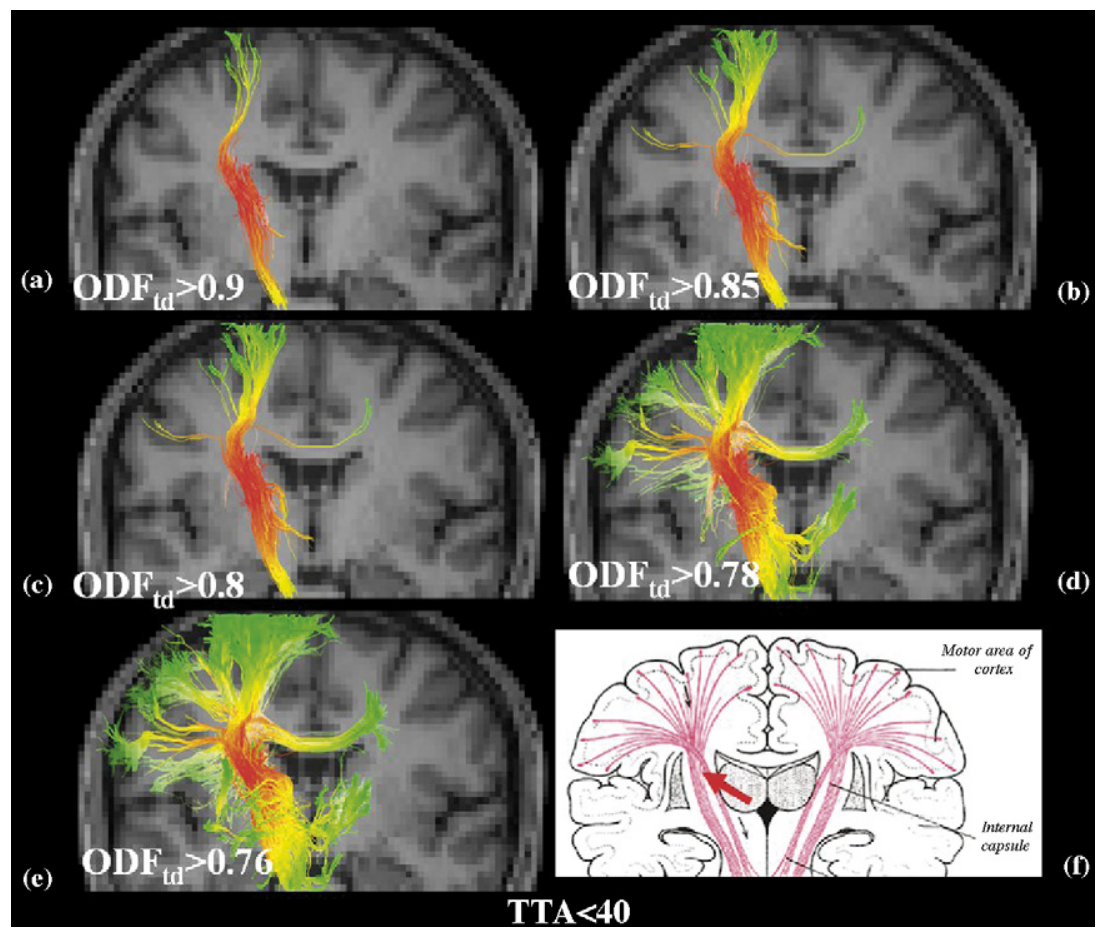

Fig. 4. Comparison between fiber propagation using the QBI-MFACT method with various $\mathrm{ODF}_{\mathrm{td}}$ values. Seed VOI was defined in the region of the internal capsule indicated by the red arrow (f). Stop criterion TTA of 40 degrees and ODF $_{\mathrm{td}}$ of $0.9-0.76$ were studied (a-e). As illustrated, tracts were consistent with sketched anatomy when $\mathrm{ODF}_{\mathrm{td}}$ was less than 0.8 , despite the fact that false-positive tracts may have been simultaneously created.

tion. Fig. 4 shows the connectivity map propagated from the defined VOI with various $\mathrm{ODF}_{\mathrm{td}}$ values. Tracts were colorcoded, ranging from red to green, according to the distances between the seed point and the traced points. Obviously, the complexity of connectivity increased while the $\mathrm{ODF}_{\mathrm{td}}$ value decreased from 0.9 to 0.76 . The results were consistent with known anatomy (Fig. 4f). From selected VOI at the internal capsule, tracts propagated to the bilateral hemisphere through the corpus callosum, to the motor cortex through the centrum semiovale, to the frontal and parietal lobes following the superior longitudinal fasciculus, and to the pons through the corticospinal tracts. All image post-processing was devel- oped in-house using c++. A laptop computer with an Intel Pentium M processor 760, 2G of memory, and Microsoft XP operating system was used. The time it took to trace these tracts, which are presented in Fig. 4, was about $10 \mathrm{~s}$.

In order to compare propagated tracts from DTI with the tracts from QBI, the same VOI of the internal capsule was adopted. The FA of 0.2 and $\mathrm{ODF}_{\mathrm{td}}$ of 0.85 were defined as the tract stopping criteria for DTI and QBI respectively. The TTA value of 60 degrees was used to yield larger tracking propagation tolerance. Fig. 5 shows the tracking results of the two approaches. The blue arrow indicates the selected seed VOI in the internal capsule. Under a

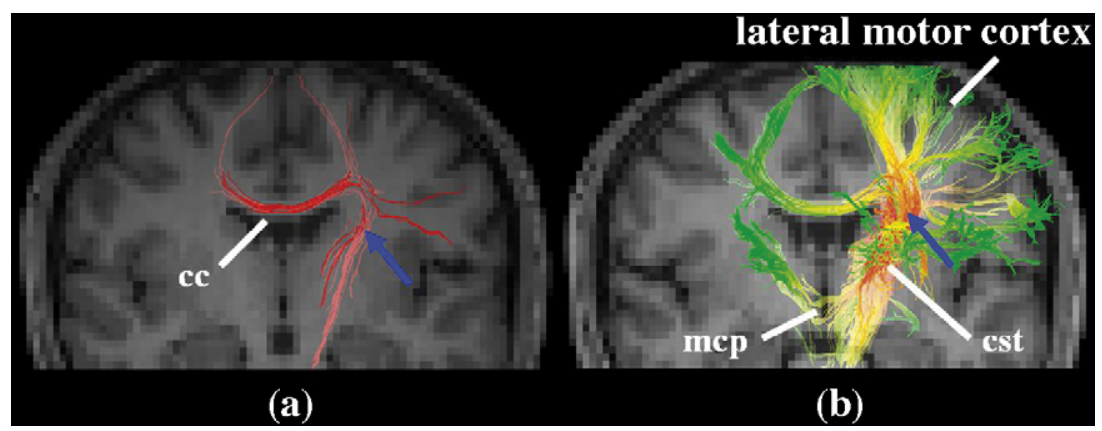

Fig. 5. Fiber tracts propagated from DTI-FACT and QBI-MFACT. The blue arrow indicates the seed VOI at the internal capsule. Tracking results using the DTI-FACT method with loose FA of 0.2 and TTA of 60 degrees showed only a few motor tracts (a). On the contrary, distinct motor pathways were extracted using the QBI-MFACT method with $\mathrm{ODF}_{\mathrm{td}}$ of 0.85 and TTA of 60 degrees. cst: corticospinal tract; mcp: middle cerebellar peduncle. 


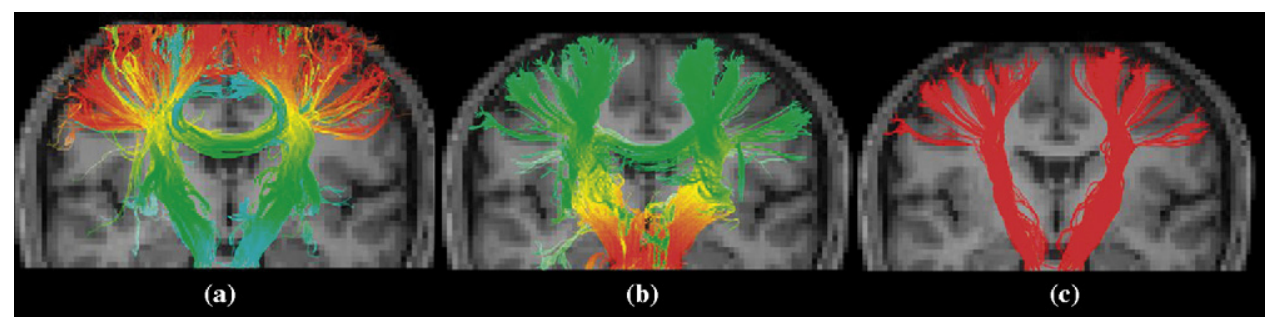

Fig. 6. VOIs of BA4 and pons for motor pathway propagation. Tracts were propagated from selected VOI at BA4 (a) and pons (b). By integrating both propagated tracts using Tract Analyzer with the Boolean function "AND", a more concrete tract was identified with a so-called brute-force fiber searching approach (c).

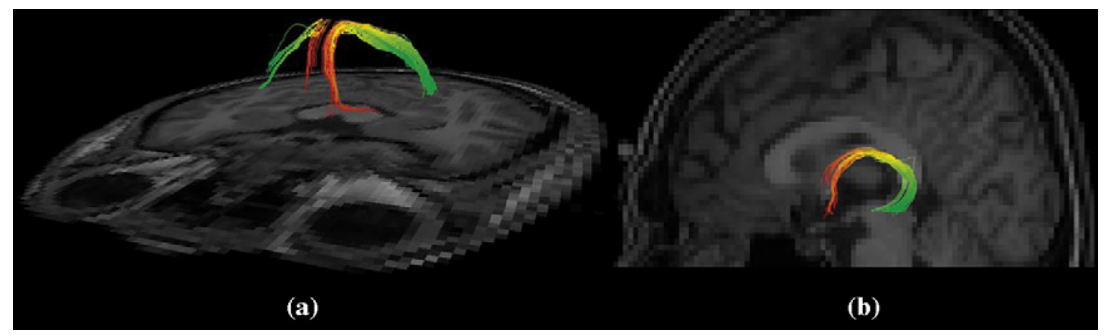

Fig. 7. QBI-MFACT tractography of the fornix in human brain. With two VOIs of the optic chiasm and geniculate nucleus, and the brute-force fiber searching approach, the fornix was identified in axial (a) and sagittal views (b).

loose stop criteria, tracts propagated from QBI with the MFACT method (Fig. 5b) revealed more concrete motor pathways than those from DTI using the FACT method (Fig. 5 a).

In order to understand the effect of VOI selection, two seed VOIs were defined in the pons and Brodmann Area 4 (BA 4, primary motor cortex). The MFACT method with TTA of 45 degrees and $\mathrm{ODF}_{\mathrm{td}}$ of 0.8 showed tracts to propagate from the two VOIs separately. Fig. 6(a) and (b) shows the neural tracts traced from the BA4 and the pons respectively. Integrating the two results with the Tract Analyzer software using the Boolean function "AND" showed a concrete motor pathway as the known anatomy (Fig. 6c). Using the same approach, the fornix was extracted with two defined VOIs in the optic chiasm and lateral geniculate nucleus, respectively (Fig. 7).

\section{Discussion}

Using information adopted from QBI regarding fiber crossing, we proposed a novel tractography algorithm MFACT to reveal the dispersion of complex fiber trajectories that have previously been evaluated by traditional tracing techniques and histological dissection. Modified from FACT [2], this method was able to detect all possible directions and has the potential to extract the anatomical connectivity in brain areas with complex neuronal connectivity. A 3D browser with a VOI selection function for various background images was designed to reveal brain markers and served as a platform for fiber tracking visualization. With Boolean functions such as AND, OR and NOT, specific neu- ronal tracts in the brain could easily be targeted and visualized [22].

Instead of using the first eigenvector information, ODF from QBI was used to map multiple fibers within each MR voxel. QBI, adopted from the q-space imaging method, demonstrated the ability to resolve fibers intercrossing. As shown in Fig. 3, the centrum semiovale is a brain area with three elements intersecting, including the corpus callosum, the corticospinal tracts and the superior longitudinal fasciculus. ODF could reflect this multi-fiber populated brain area. Motor pathways from the cerebral peduncle through to the internal capsule and centrum semiovale and to the cerebral cortex were easily identified with VOI at the internal capsule (Fig. 4), BA4, or pons (Fig. 6). The results were more consistent with known anatomy in comparison to tracts from DTI.

Our results may be regarded as a kind of tree structure. The tracts initiated from a selected seed point (root) and spread along any possible orientation of ODF until the next node. The extended branch of nodes was defined as a new root and repeatedly propagated until one of the stop criteria was met. Finally, a tree structure pathway could be identified. This method is advantageous for mapping all possible tracts within the brain from user-defined regions. However, it may generate lots of complicated and unwarranted tracts which are difficult verified and interpreted. Such false-positive tracts were also found in other MFT approaches [5,18]. In order to avoid such errors, it is important to extract the expected tracts by selecting multiple VOIs with reference to anatomical templates, brain markers, and Boolean functions. 
Notably, the brute-force fiber searching approach might help to identify valid tracts in our study [22]. As the results in Fig. 6 show, tracts generated from VOIs at the pons or BA4 unfold specific motor pathways. Both propagated tracts revealed motor pathways accurately while the VOI of BA4 showed more concrete tracts between the cerebral cortex than that from the pons (Fig. 6a). Relatively, VOI at the pons revealed more tracts into the cerebellum (Fig. 6b). These results imply that VOI should be better defined close to the region of interest to acquire accurate information. Furthermore, the expected motor pathways could be extracted by integration of pathways from the pons to BA4 and from BA4 to the pons. This means that anticipated connections between two regions should be identified by deriving the pathways that include tracking results from region $\mathrm{A}$ to region $\mathrm{B}$ and from region $\mathrm{B}$ to region $\mathrm{A}$. This concept has not been previously applied in MFT methods due to complexity and heavy computation loading. Despite the fact that this type of tract calculation is time consuming, our results showed that it might help in defining tracts accurately.

Although our method has the potential to reveal most possible tracts, selection of stop criteria is critical to the results. Two stop criteria, TTA and $\mathrm{ODF}_{\mathrm{td}}$, were adopted in this study to regulate the generation of pathway trajectories and to prune incorrect neuronal branches. TTA was used to define the joinable range of neuronal projections toward adjacent voxels. This was crucial to the expected curvature of fiber tracts by defining the appropriate value of TTA. Previous reports of DTI tractography had values of 30-70 degrees [22,24-26]. This was in agreement with the fact that there are no high curvature fiber bundles in the human brain and the selection of this value depends on the imaging voxel size. Despite these common convictions, standard values have not been established due to the lack of a gold standard. In this study, the default value of TTA was set to 50 degrees. Users could define the TTA value depending on the voxel size, SNR, and prior knowledge. Another stop criterion was the threshold length of ODF, $\mathrm{ODF}_{\mathrm{td}}$. As shown in Fig. 4, due to extra diffusion directions being identified within each MR voxel, more neural tracts were revealed under looser $\mathrm{ODF}_{\mathrm{td}}$ restraint. On the contrary, fewer tracts were mapped with stricter selection of $\mathrm{ODF}_{\mathrm{td}}$ values. This $\mathrm{ODF}_{\mathrm{td}}$ can serve as a criterion for pruning erroneous vectors that come from MR noise or partial volume effect. Though the standard threshold value for this ODF length is still lacking, MR voxels with diffusion directions $\geqq 3$ represented more than $33 \%$ of the whole brain under the $\mathrm{ODF}_{\mathrm{td}}$ of 0.8 in our study. This result indicated that the use of only two or three major fiber directions for fiber tracking may be inadequate to map all possible fiber tracts in the human brain $[5,18]$. Despite the time and memory consumption required to calculate the propagation of fiber pathways, using a greater number of directions to describe the diffusion ODF pattern in QBI would provide more accurate orientations of major fibers by decreasing small angular separation. These limitations can be further solved by using a high-level computer or a distributed process in the pc-cluster. In addition, MFACT is not only applicable in QBI, but also in other diffusion imaging approaches that provide multiple fiber orientations within each voxel.

The fornix has previously been hard to detect because of its adjacent location to cerebral-spinal fluid (CSF) spaces, i.e. the ventricles and interhemispheric fissure. The measured DTI pattern and tract delineation may partially be affected by rapid, isotropically diffusing CSF volume. Here, by selecting VOIs at the optic chiasm and LGN, the fornix was identified in this study (Fig. 7). Although our results showed that the QBI approach appeared suitable for providing information regarding multiple components within a voxel, current understanding of the relationship between the observed diffusion signal and properties of fiber populations failed to be described completely [18]. Partial volume effects inevitably influence the results of any diffusion imaging technique. From present results, it is still difficult to identify the relationship between adjacent fibers, i.e. whether they are crossing, kissing, diverging, or converging. However, we believe that further improvement of the MFACT algorithm, with data acquisition involving a higher spatial resolution and advanced diffusion imaging approaches, will increase the level of confidence for mapping cerebral connections.

In conclusion, a novel tracking algorithm, MFACT, was developed to map intricate neural pathways non-invasively and the propagated connectivity exhibited promising potential in exploring the human brain. The tracking process was performed in a shorter time and could be applied to most diffusion imaging approaches with multiple discrete fiber distributions. In this study, using the MFACT algorithm with QBI data from human brain, the well-known fiber tract dispersions from the internal capsule, cortical spinal tracts, and fornix were precisely reconstructed with appropriate criteria of TTA and $\mathrm{ODF}_{\mathrm{td}}$. This accomplishment demonstrates the potential of MFACT in revealing anatomical or functional connectivity for neuroscientific study and in clinical applications.

\section{Acknowledgements}

The work was supported in part by research grants from National Science Council (NSC- 96-2752-H-010-004-PAE) and Taipei City Hospital (TCH-95002-62-071).

\section{Conflict of interest}

To my knowledge, all of my possible conflicts of interest and those of my coauthors, financial or otherwise, related directly or indirectly to this work are clearly and completely indicated, and all grant or other financial support, as well as any material support, is listed in the appropriate sections of this manuscript. 


\section{References}

[1] Basser PJ, Pajevic S, Pierpaoli C, Duda J, Aldroubi A. In vivo fiber tractography using DT-MRI data. Magn Reson Med 2000;44(4):625-32.

[2] Mori S, Crain BJ, Chacko VP, van Zijl PC. Three-dimensional tracking of axonal projections in the brain by magnetic resonance imaging. Ann Neurol 1999;45(2):265-9.

[3] Conturo TE, Lori NF, Cull TS, Akbudak E, Snyder AZ, Shimony JS, et al. Tracking neuronal fiber pathways in the living human brain. Proc Natl Acad Sci USA 1999;96(18):10422-7.

[4] Catani M, Howard RJ, Pajevic S, Jones DK. Virtual in vivo interactive dissection of white matter fasciculi in the human brain. Neuroimage 2002;17(1):77-94.

[5] Behrens TE, Berg HJ, Jbabdi S, Rushworth MF, Woolrich MW. Probabilistic diffusion tractography with multiple fibre orientations: what can we gain? Neuroimage 2007;34(1):144-55.

[6] Wedeen VJ, Hagmann P, Tseng WY, Reese TG, Weisskoff RM. Mapping complex tissue architecture with diffusion spectrum magnetic resonance imaging. Magn Reson Med 2005;54(6):1377-86.

[7] Tuch DS. Q-ball imaging. Magn Reson Med 2004;52(6):1358-72.

[8] Assaf Y, Freidlin RZ, Rohde GK, Basser PJ. New modeling and experimental framework to characterize hindered and restricted water diffusion in brain white matter. Magn Reson Med 2004;52(5):965-78.

[9] Alexander DC. Multiple-fiber reconstruction algorithms for diffusion MRI. Ann N Y Acad Sci 2005;1064:113-33.

[10] Jansons KM, Alexander DC. Persistent Angular Structure: new insights from diffusion MRI data. Dummy version. Inf Proc Med Imag 2003;18:672-83.

[11] Tournier JD, Calamante F, Connelly A. Robust determination of the fibre orientation distribution in diffusion MRI: non-negativity constrained super-resolved spherical deconvolution. Neuroimage 2007.

[12] Flavio DA, Giovanna R, Paola S, Rafael Alonso C, Giuseppe S, Ferruccio F. A model-based deconvolution approach to solve fiber crossing in diffusion-weighted MR imaging. IEEE Trans Biomed Eng 2007;54(3):462-72.

[13] Tuch DS, Reese TG, Wiegell MR, Makris N, Belliveau JW, Wedeen VJ. High angular resolution diffusion imaging reveals intravoxel white matter fiber heterogeneity. Magn Reson Med 2002;48(4):577-82.

[14] Hosey T, Williams G, Ansorge R. Inference of multiple fiber orientations in high angular resolution diffusion imaging. Magn Reson Med 2005;54(6):1480-9.
[15] Anderson AW. Measurement of fiber orientation distributions using high angular resolution diffusion imaging. Magn Reson Med 2005;54(5):1194-206.

[16] Campbell JS, Siddiqi K, Rymar VV, Sadikot AF, Pike GB. Flow-based fiber tracking with diffusion tensor and Q-ball data: validation and comparison to principal diffusion direction techniques. Neuroimage 2005;27(4):725-36

[17] Pichon E, Westin CF, Tannenbaum AR. A Hamilton-Jacobi-Bellman approach to high angular resolution diffusion tractography. Med Image Comput Comput Assist Interv Int Conf Med Image Comput Comput Assist Interv 2005;8(Pt 1):180-7.

[18] Parker GJ, Alexander DC. Probabilistic anatomical connectivity derived from the microscopic persistent angular structure of cerebral tissue. Philos Trans R Soc Lond B Biol Sci 2005;360(1457): 893-902.

[19] Cho KH, Chao YP, Li YC, Chen JH, Lin CP. Mapping fiber density distribution with diffusion spectrum imaging. In: 13th Scientific Meeting \& Exhibition of International Society for Magnetic Resonance in Medicine. 2005. p. 577

[20] Xue R, van Zijl PC, Crain BJ, Solaiyappan M, Mori S. In vivo threedimensional reconstruction of rat brain axonal projections by diffusion tensor imaging. Magn Reson Med 1999;42(6):1123-7.

[21] Huang H, Zhang J, van Zijl PC, Mori S. Analysis of noise effects on DTI-based tractography using the brute-force and multi-ROI approach. Magn Reson Med 2004;52(3):559-65.

[22] Jiang H, van Zijl PC, Kim J, Pearlson GD, Mori S. DtiStudio: resource program for diffusion tensor computation and fiber bundle tracking. Comput Methods Programs Biomed 2006;81(2):106-16.

[23] Masutani Y, Aoki S, Abe O, Hayashi N, Otomo K. MR diffusion tensor imaging: recent advance and new techniques for diffusion tensor visualization. Eur J Radiol 2003;46(1):53-66.

[24] Cheng P, Magnotta VA, Wu D, Nopoulos P, Moser DJ, Paulsen $\mathrm{J}$, et al. Evaluation of the GTRACT diffusion tensor tractography algorithm: a validation and reliability study. Neuroimage 2006;31(3): 1075-85.

[25] Kreher BW, Schneider JF, Mader I, Martin E, Hennig J, Il'yasov KA. Multitensor approach for analysis and tracking of complex fiber configurations. Magn Reson Med 2005;54(5):1216-25.

[26] Nimsky C, Ganslandt O, Fahlbusch R. Implementation of fiber tract navigation. Neurosurgery 2006;58(4 Suppl. 2) [ONS-292-303; discussion ONS-303-294]. 